\title{
On poor and not so poor thought experiments. A reply to Daniel Cohnitz
}

\author{
Jeanne Peijnenburg • David Atkinson
}

Received: 15 January 2007 / Accepted: 15 January 2007 / Published online: 22 May 2007

(C) Springer Science+Business Media B.V. 2007

We have never entirely agreed with Daniel Cohnitz on the status and rôle of thought experiments. Several years ago, enjoying a splendid lunch together in the city of Ghent, we cheerfully agreed to disagree on the matter; and now that Cohnitz has published his considered opinion of our views, we are glad that we have the opportunity to write a rejoinder and to explicate some of our disagreements. We choose not to deal here with all the issues that Cohnitz raises, but rather to restrict ourselves to three specific points.

The first point concerns the way in which Cohnitz represents our views. He summarizes them in five theses, (PA1)-(PA5), and while we have no dispute with the wording of (PA1)-(PA4), we consider (PA5) to be somewhat misleading. Putting into our mouths the declamation that "In philosophy we cannot turn to crucial experiments (whereas in science we can)" wrongly suggests that we take crucial experiments to constitute the pivotal difference between philosophy and science. At no place do we claim that disputes over thought experiments in physics are always settled by carrying out a crucial experiment. Cohnitz writes: "As Peijnenburg and Atkinson seem to admit, this [the settling by a crucial experiment] is not the case for all physical thought experiments." Seem to admit? Nay, we roundly asseverate the fact! After that sentence, Cohnitz continues as follows:

“It seems clear, for example in Galileo's Pisa-experiment that an empirical test could not have solved the matter the way this thought experiment did. But this point is not very remarkable, given that Peijnenburg and Atkinson argue elsewhere that Galileo's Pisa-argument was a poor one in the first place (Atkinson and Peijnenburg 2004)". (Cohnitz 2006, p. 384).

Each of these two sentences is misleading. Contrary to what Cohnitz claims, Galileo's Pisastory is one of the few thought experiments that can be settled by a real experiment. Take

\footnotetext{
J. Peijnenburg $(\varangle)$. D. Atkinson

Faculty of Philosophy, University of Groningen, Groningen, The Netherlands

e-mail: jeanne.peijnenburg@rug.nl
}

D. Atkinson

e-mail: d.atkinson@rug.nl 
two balls of lead, the one ball ten times as heavy as the other. Drop the heavy ball from a tower and let us assume that it takes $5 \mathrm{~s}$ to reach the ground. Now take the lighter ball and drop it from the tower in the same way. According to Aristotle's theory, it will take $50 \mathrm{~s}$ for the lighter ball to reach the ground. This is a prediction that can easily be falsified in a real experiment, even if there are disturbing influences of wind, air friction and the like. In fact, it was falsified by the Belgian engineer and mathematician Simon Stevin as early as 1605 . Stevin performed the experiment by letting the balls fall from a height of 30 feet down on to a plank. He noticed that the light ball fell towards the plank, not ten times more slowly than the other ball, "but so equally with the other that the sound of the two in striking will seem to come back as one single report" (Stevin in De staticae, quoted in Atkinson and Peijnenburg, 2004, p. 135). All this is of course in complete accordance with Galileo's attack on Aristotle.

It is also misleading to state that we "argue elsewhere that Galileo's Pisa-argument was a poor one (Atkinson and Peijnenburg 2004)". As a destructive thought experiment, refuting the Aristotelian theory of falling bodies, we deem Galileo's thought experiment to be unparalleled, one of the best. But as a constructive thought experiment, claiming that all bodies fall at the same rate, it has a serious flaw. For it fails to make explicit a hidden assumption that is not always applicable, namely that the rate of fall of a body depends only on its weight, and on nothing else. In our paper of 2004 we describe various empirical situations, in some of which Aristotle's theory holds, and others where Galileo's claim is true. We then conclude that the best way of developing a theory of falling bodies is by doing real experiments. None of this is to say that Galileo's Pisa-argument is poor in the sense of the criteria that we formulated in Peijnenburg and Atkinson (2003). It is not. The Pisa-argument does not trigger conflicting intuitions, nor does it give rise to question-begging conclusions.

The second point we address concerns the analysis of Newton's bucket and Einstein's spheroid. In the 2003 paper we argued that these two thought experiments are in essence the same, but that Einstein and Newton drew opposite conclusions from it. Cohnitz disagrees. He does not object to our claim that the two thought experiments basically deal with the same problem, and thus that Einstein's question 'Why is the one fluid body a sphere and the other an ellipsoid?' is basically the same as Newton's question 'Why is the water surface plane at the beginning of the experiment and concave at the end?'. Rather, he has a different opinion about how to characterize Newton's and Einstein's answers to this problem. Whereas we portray these answers as contradictory conclusions drawn from the same thought experiment, Cohnitz characterizes them as "competing alternative hypotheses that are empirically equivalent”. According to Cohnitz:

"The difference between Einstein and Newton is not a difference in intuitions concerning the details of the case imagined, but the question what would serve as the best explanation for such a phenomenon. This is in no way a special problem of thought experimentation, but the old problem of how to decide between empirically equivalent competing hypotheses" (Cohnitz 2006, p. 382).

We agree with Cohnitz that the conflict between Newton and Einstein can be seen as a clash between two explanatory hypotheses. However, our point is that in this case the hypotheses are empty: nothing empirical whatsoever follows from them. For not only does Newton's principle of absolute space lack empirical content, the same goes for Einstein's idea that centrifugal forces are caused by matter in the rest of the universe, also known as Mach's Principle. Einstein tried to make this principle part of his theory of general relativity, but later in his life he admitted that this attempt had failed. Mach's Principle was never more than an inessential ornament, to be added or removed without empirical consequence. Hence it is 
nugatory to talk here, as Cohnitz does, about hypotheses that are "empirically equivalent". The hypotheses of Newton and Einstein are indeed equivalent, but they are not empirically equivalent, since they have no empirical content at all.

The third and last point touches the status of philosophical thought experiments, in particular Jackson's Mary and Chalmers' zombie. Cohnitz challenges our claim that these thought experiments trigger conflicting intuitions. He argues that in both cases the intuitions are shared: we all have the intuition that Mary learns something new, and we all share the intuition that zombies are conceivable. This is not to say that there is no diagreement. Cohnitz argues that philosophers do disagree, not on these shared intuitions as such, but on what follows from them:

"The intuition ('Mary learned something new') is shared, what is doubted is what conclusions are to be drawn from it. [.....] it is generally agreed that the existence of zombies is such a logical possibility ... The question is what follows from this shared intuition" (Cohnitz 2006, p. 386-387).

Apparently Cohnitz's view is that all philosophers have the same intuition about Jackson's Mary, and that they have the same intuition about Chalmers' zombie; they just diverge widely on the consequences of these joint intuitions. But if this is indeed Cohnitz's considered opinion, we fail to discern a significant difference between his and our points of view. If some philosophers believe that consequence $\mathrm{C}$ follows from intuition I, while other philosophers believe that not-C follows from I, does this not mean that the philosophers have a conflict over I itself? And is this not very close to saying that they have conflicting intuitions? Perhaps Daniel Cohnitz will in the not too distant future agree with us on this matter, hopefully while savouring yet another gustatory excellence.

\section{References}

Atkinson, D., \& Peijnenburg, J. (2004). Galileo and prior philosophy. Studies in History and Philosophy of Science, 35, 115-136.

Cohnitz, D. (2006). Poor thought experiments? A comment on Peijnenburg and Atkinson. Journal for General Philosophy of Science, 37(2), 373-392.

Peijnenburg, J., \& Atkinson, D. (2003). When are thought experiments poor ones? Journal for General Philosophy of Science, 34, 305-332. 